\title{
Multiscale Principal Component Analysis to Predict Atrial Fibrillation Reversion to Sinus Rhythm
}

\author{
Raquel Cervigón ${ }^{1}$, Javier Moreno² ${ }^{2}$ José Millet $^{4}$, Francisco Castells ${ }^{4}$ \\ ${ }^{1}$ Universidad de Castilla-La Mancha, Cuenca, Spain \\ ${ }^{2}$ Arrhythmia Unit. Hospital Ramon y Cajal, Madrid. Ctra. de Colmenar Viejo, Spain \\ ${ }^{3}$ Universidad Politécnica de Valencia, Valencia, Spain
}

\begin{abstract}
The objective of this study was to evaluate potential of Multiscale principal Analysis (MSPCA) to extract information of atrial electrograms as predictors of atrial fibrillation (AF) recurrence after ablation. Database is composed of intra-atrial recordings from 43 AF patients that were registered previous to an ablation procedure. MSPCA was applied to each atrium and main frequency and entropy were determined. We could find differences in main frequency in the left atrium between patients from both groups. Moreover, patients that maintenance sinus rhythm group showed a dominant atrial frequency trend from right to left atrium. In addition, non-recurrent $A F$ group showed an enhancement of differences of regularity between both atria. These differences show the efficiency of MSPCA to characterize cardiac dynamic. High frequency values and not differences in main atrial frequency along the right atrium may indicate that $A F$ reversion to sinus rhythm is more difficult.
\end{abstract}

\section{Introduction}

Atrial fibrillation (AF) is the most frequent arrhythmia. Prevalence increased from $0.1 \%$ among adults younger $\tan 55$ years to $9.0 \%$ in persons aged 80 years or older [1]. The electrophysiological mechanism during AF involves the presence of multiple simultaneous atrial wavefronts re-entry circulating continuously and chaotically around the atria, whose foci in most cases are found in the pulmonary veins.

Pulmonary vein isolation is increasingly being used for its efficacy compared with antiarrhythmic drugs. The use of radiofrequency energy destroy this small area of tissue [2]. The resulting tissue blocks the extra electrical signals from the pulmonary veins reaching the left atrium, so the area can no longer generate or conduct the fast, irregular impulses. This process is repeated around the opening of each of the four pulmonary veins. The long-term goal of this procedure is to eliminate the need for medications to prevent AF.
Over the past years, this technique has evolved from being an experimental procedure to the most common ablation performed in many electrophysiology laboratories throughout the world. Nevertheless, many aspects of the therapy are still controversial, patient management, prevention and treatment of complications and the most important, success rate, because at the moment it is difficult to predict when AF will be cured.

In this study, we analyse the recurrence outcome of paroxysmal and persistent AF patients in a three months follow-up. To perform this analysis, we propose a method to extract the main activity of the atria, with the application of multiscale principal component analysis to atrial electrograms, atrial activity regularity and dominant frequency from atrial activity from dipoles located along the left and the right atria.

\section{Materials}

$\mathrm{AF}$ intracardiac recordings were registered in 43 patients immediately before AF ablation procedure. Table 1 shows different parameters from recurrent and nonrecurrent AF patients where there was no statistically significant difference between groups. A 24-pole catheter (Orbiter, Bard Electrophysiology, 2-9-2 mm electrode spacing) was inserted through the femoral vein and positioned in the right atrium (RA) with the distal dipoles into the coronary sinus (CS) to record left atrial (LA) electrical activity as well. The medium and proximal group of electrodes were located spanning the RA freewall peritricuspid area, from the coronary sinus ostium to the upper part of the inter-atrial region. Using this catheter, 12 bipolar intracardiac electrograms from the RA (dipoles from 15-16 to 21-22) and LA (dipoles 1-2, 34, 5-6 and 7-8), were digitally recorded at $1 \mathrm{kHz}$ sampling rate (16 bit A/D conversion; Polygraph Prucka CardioLab, General Electric). Fifty to 60 seconds recordings from paroxysmal and persistent $\mathrm{AF}$ patients were analysed. All patients were monitored after ablation, and were divided in 2 groups according to AF recurrence outcome 3 months after ablation procedure. 
Table 1. Patient Clinical Characteristics

\begin{tabular}{lll}
\hline Parameters & $\begin{array}{l}\text { Recurrent } \\
\text { AF }\end{array}$ & $\begin{array}{l}\text { Non- } \\
\text { recurrent AF }\end{array}$ \\
\hline Paroxysmal AF & 10 & 13 \\
Persistent AF & 7 & 13 \\
Male (\%) & $11(65 \%)$ & $24(88 \%)$ \\
Age (years) & $51 \pm 14$ & $48 \pm 14$ \\
Structural heart disease (\%) & $4(29 \%)$ & $5(22 \%)$ \\
Left atrium size (mm) & $44 \pm 8$ & $44 \pm 6$ \\
\hline
\end{tabular}

\section{Methods}

Multiscale Principal Component Analysis (MSPCA) combines the ability of Principal Component Analysis (PCA) to reconstruct, starting from a multivariate signal and using a simple representation at each resolution level, a simplified multivariate signal. PCA transforms an $\mathrm{n} \mathrm{x} \mathrm{m}$ data matrix, $\mathbf{X}$ by combining the variables as a linear weighted sum as,

$$
\mathrm{X}=\mathrm{TP}^{\mathrm{T}}
$$

where, $\mathbf{P}$ are the principal component loadings, $\mathbf{T}$ are the principal component scores, $\mathrm{n}$ and $\mathrm{m}$ are the number of measurements and variables respectively.

Wavelets are a family of basis functions that are localized in both time and frequency. To combine the benefits of PCA and wavelets, the measurements for each variable, in our case, for each chamber chanel, are decomposed to the wavelet coefficients using the same orthonormal wavelet for each variable. This results in transformation of the data matrix, $\mathbf{X}$, into a matrix $\mathbf{W X}$, where $\mathbf{W}$ is an $n$ $\mathrm{x} \mathrm{n}$ orthonormal matrix representing the orthonormal wavelet transformation operator containing the filter coefficients, it produces $k$ th wavelet coefficient at $j$ th subband as $\mathrm{wj}_{\mathrm{j}, \mathrm{k} .} L$ level decomposition results in $L$ th approximation subband $c \mathrm{AL}$ and $j$ th detail subband $c \mathrm{Dj}$, where $\mathrm{j}=1,2, \ldots, \mathrm{L}$. M number of electrogram channels in each chamber are wavelet decomposed and $j$ th subband of all channels are collected in a matrix, to form a wavelet subband matrix. This results in " $\mathrm{L}+1$ " subband matrices. Multiscale wavelet subband matrix for approximation band is $A_{L}$ and subband matrices for details are $D_{j}$, where $\mathrm{j}=1,2, \ldots, \mathrm{L}$. In this formulation, the number of columns is the number of electrogram channels and number of rows is the number of coefficients in that particular subband. In our study, Daubechies 4 was selected as mother wavelet on the decomposition proccess, for 4 lead intracardiac electrograms in each chamber and for five levels wavelet decomposition.

MSPCA generalizes the normal PCA of a multivariate signal represented as a matrix by performing PCA of the wavelet coefficients at each scale, followed by combining the results at relevant scales, in this approach the relevant scale was the highest order subband that contains the main atrial activity.

This approach is equivalent to adaptively filtering each value of the scores and residuals by a filter of dyadic length that is best suited for separating the deterministic change from the normal process variation. The detection limits for the scores and residuals also adapt to the nature of the signal, in this case only the first component was chosen.

\subsection{Data analysis}

Two different parameters were estimated, dominant frequency and regularity of the signal. Welch's modified periodogram method was used to estimate the main or dominant frequency. It was identified as the frequency relating to the maximum amplitude in the frequency band between 3 and $10 \mathrm{~Hz}$. Moreover, Shannon Entropy was calculated to analyse the disorder of the extracted signals [5]. As a final step statistical techniques were applied to the extracted parameters. The parameters are expressed in terms of mean and standard deviation values. Independent t-student tests were used for comparison between both groups of results. Results were considered to be statistically significant at $\mathrm{p}<0.05$.

\section{Results}

MSPCA preprocessing extracts the main activations. In figure 1 , it is possible to observe an example of the extracted signal after MSPCA application to a dipole located in the right atrium.

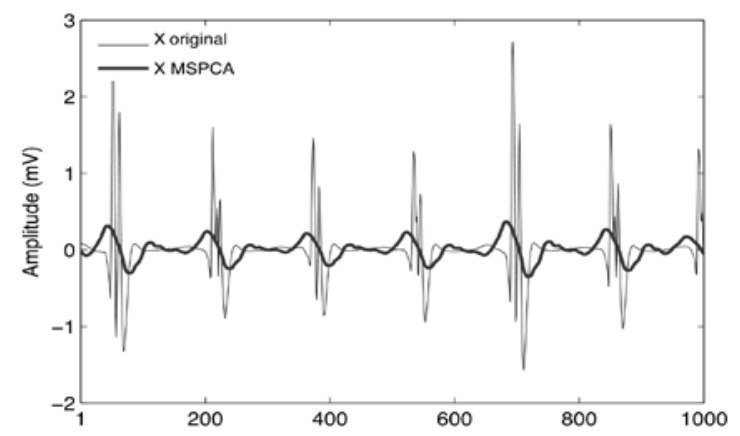

Figure 1. Atrial signal from the RA and after MSPCA application during 1 second.

Atrial activity extracted from MSPCA showed statistical significant differences $(p=0.0287)$ between in the dominant frequency from dipoles located in the left chamber, with $5.46 \pm 1.26 \mathrm{~Hz}$ in patients without recurrences in the arrhythmia and $6.37 \pm 1.29 \mathrm{~Hz}$ in the patients with AF recurrence (Figure 2). Moreover, not changes were found the right atrium dominant frequency between sinus rhythm maintenance and $\mathrm{AF}$ recurrence in patients, $6.27 \pm 1.06 \mathrm{~Hz}$ vs. $6.72 \pm 0.95 \mathrm{~Hz}(\mathrm{p}=0.169)$. 
Moreover, dominant atrial frequency values exhibited a RA to LA gradient, with lower values at the LA with $5.47 \pm 1.26 \mathrm{~Hz}$ vs. $6.27 \pm 1.06 \mathrm{~Hz}$ in the RA in the patients that maintenance sinus rhythm and these differences were statistically significant ( $\mathrm{p}=0.004)$ (Figure 3 ).

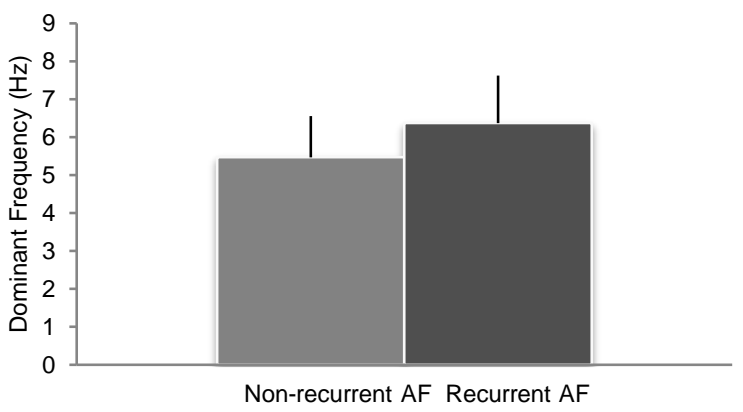

Figure 2. Left atrium dominant frequency in nonrecurrent AF (light grey) and recurrent AF (dark grey).

Nevertheless, this gradient was not shown significant differences in patients with recurrences in the arrhythmia with $6.37 \pm 1.29 \mathrm{~Hz}$ in the LA vs. $6.72 \pm 0.95 \mathrm{~Hz}$ in the RA $(\mathrm{p}=0.337)$.

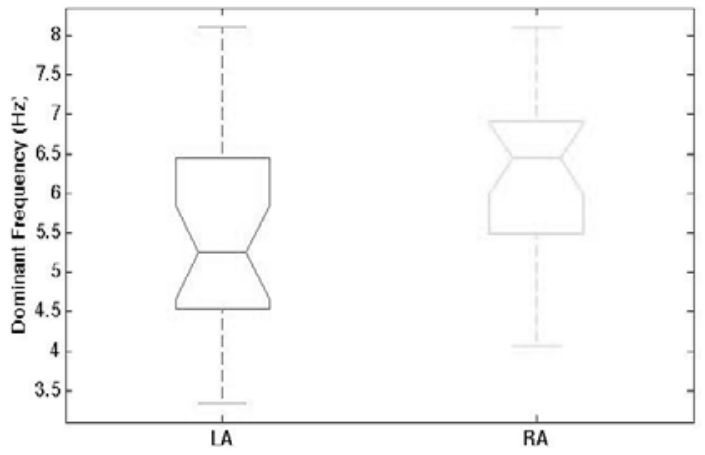

Figure 3. Dominant Atrial frequency trend in nonrecurrent AF group.

In addition, it was possible to find difference between along the atria in the regularity of the signals after MSPCA application. These differences were higher in the group with maintenance in sinus rhythm that in patients with recurrences in the arrhythmia.

In the patients with recurrence in the arrhythmia, shannon entropy was higher in the RA $52.53 \pm 34.22$ than in the LA, 30.23 \pm 25.06 , with statistical significant differences, $\mathrm{p}=0.004$ (Figure 4).

In the group with maintenance sinus rhythm in the arrhythmia, wavelet shannon entropy was higher in the RA with $59.87 \pm 32.49$ than in the LA with $27.09 \pm 14.27$, these differences were statistical significant, $\mathrm{p}<0.001$ (Figure 5).

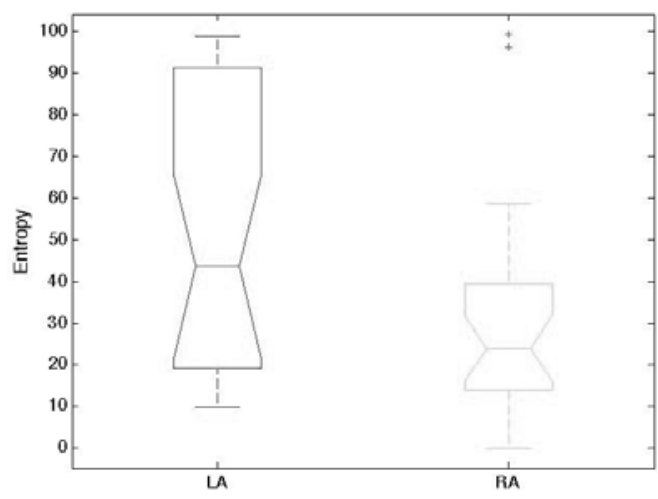

Figure 4. Entropy differences between both atria in the patients with recurrences in the arrhytmia.

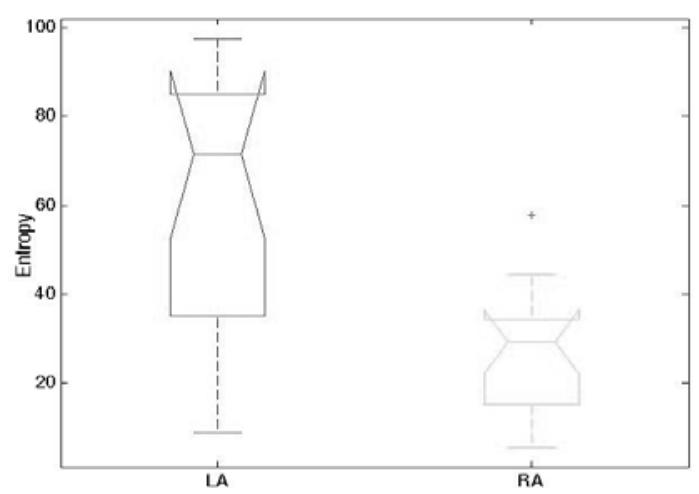

Figure 5. Wavelet entropy differences between both atria in the non-recurrent AF group.

\section{Discussion}

MSPCA is appropriate for modelling of data continuing contributions from events that changes over time and frequency, by selecting the numbers of retained principal components. MSPCA has already been successfully implemented in biomedical studies, such as multichannel ECG data compression [6] or in milling tool wear monitoring method [7]. Moreover, MSPCA has been introduced for multivariate statistical process control [8] and it has been also used for real process fault detection and diagnosis $[9,10]$.

MSPCA can be a useful to extract and characterize the main activity of both atria in order to provide some predictive information regarding the recurrence outcome after 3-months follow-up.

Predictors of AF ablation success and AF recurrence are currently of high clinical interest. On multivariate analysis, a shorter ablation duration, younger age, male gender, and the presence of hypertension are predictors of 
overall clinical success included AF termination by ablation [11]. In addition, the dominant atrial frequency has been described as a predictor of AF ablation outcome, where long cycle lengths were responsible for the organization of the arrhythmia, whereas the shortest cycle lengths were related to recurrences [12]. Based on mechanistic studies, rotor sites are expected to exhibit greater electrical periodicity, unique spectral characteristics such as higher dominant frequency [13], and higher entropy [14,15], distinguishing them from other atrial sites [16]. Dominant frequency from atrial signals extracted with MSPCA showed a differences in left atrium main frequency in the left atrium with a more rapid activity in the patients with recurrences in the arrhythmia. Moreover, differences in main frequency in both atria were found in the patients that maintenance the sinus rhythm.

Certainly, the results described showed differences in shannon entropy between both atria in recurrent and nonrecurrent $\mathrm{AF}$ group, but with higher atrial gradient in the patients that maintenance sinus rhythm. Previous studies have showed that patients that remained in sinus rhythm presented different entropy values tan those who turned back to AF [14].

As result, the study shows that MSPCA is effective in AF analysis. MSPCA was able to extract main components of atrial activity time series. Nevertheless, larger studies are needed to quantify the difference distribution of components energy along the atria in both groups. Moreover, this study is consistent with previous research studies that showed that patients that remained in sinus rhythm presented a shower atrial activity in the left atrium than those who turned back to AF.

\section{Acknowledgements}

This work was supported by the Castilla-La Mancha Research Scheme (PPII-2014-024-P).

\section{References}

[1] A S Go, E M Hylek, K A Phillips, Y Chang, L E Henault, J V Selby, and D E Singer, "Prevalence of diagnosed atrial fibrillation in adults: national implications for rhythm management and stroke prevention: the anticoagulation and risk factors in atrial fibrillation (atria) study,” JAMA , vol. 285, no. 18, pp. 2370-5, May 2001.

[2] M Haissaguerre, DC Shah, and P Jais, "Electrophysiological breakthroughs from the left atrium to the pulmonary veins," Circulation, vol. 102, pp. 2463 n5, 2000.

[3] L. N. Sharma, S. Dandapat, and A.Mahanta, "ECG signal denoising using higher order statistics in Wavelet subbands,” Biomed. Signal Process. Control, Elsevier, vol. 5, pp. 214-222, 2010.

[4] L. N. Sharma, S. Dandapat, and A. Mahanta, "Kurtosisbased noise estimation multiscale energy to denoise ECG signal,” in Signal, Image and Video Processing. New York:
Springer-Verlag, 2011.

[5] Claude Elwood Shannon and Warren Weaver. "The mathematical theory of communication". University of Illinois Press, Urbana, 1998.

[6] L. N. Sharma, S. Dandapat, and Anil Mahanta, "Multichannel ECG Data Compression Based on Multiscale Principal Component Analysis". IEEE Transactions on Information Technology in Biomedicine, Vol. 16, 4, 2012

[7] G Wang, Y Zhang, C Liu, Q Xie, Y Xu. "A new tool wear monitoring method based on multi-scale PCA Journal of Intelligent Manufacturing”, 2016 - Springer

[8] B. R. Bakshi, "Multiscale PCA with application to MSPC monitoring," AIChE J., vol. 44, no. 7, pp. 1596-1610, Jul. 1998.

[9] M. Misra, H. H. Yue, S. J. Qin, and C. Ling, “Multivariate process monitoring and fault diagnosis by multi-scale PCA,” Comput. Chem. Eng., Elsevier, vol. 26, pp. 12811293, 2002.

[10] C. Rosen and J. A. Lennox, "Multivariate and multiscale monitoring of wastewater treatment operation," Wat. Res., Elsevier, vol. 35, no. 14, pp. 3402-3410, 2001.

[11] E Kevin Heist, Fadi Chalhoub, Conor Barrett, Stephan Danik, Jeremy N Ruskin, and Moussa Mansour, "Predictors of atrial fibrillation termination and clinical success of catheter ablation of persistent atrial fibrillation," Am J Cardiol , vol. 110, no. 4, pp. 545-51, Aug 2012.

[12] Yasuo Okumura, Ichiro Watanabe, Masayoshi Kofune, Koichi Nagashima, Kazumasa Sonoda, Hiroaki Mano, Kimie Ohkubo, Toshiko Nakai, and Atsushi Hirayama, "Characteristics and distribution of complex fractionated atrial electrograms and the dominant frequency during atrial fibrillation: relationship to the response and outcome of circumferential pulmonary vein isolation,” J Interv Card Electrophysiol , vol. 34, no. 3, pp. 267-75, Sep 2012.

[13] Felipe Atienza, Jesús Almendral, José Jalife, Sharon Zlochiver, Robert Ploutz-Snyder, Esteban G Torrecilla, Angel Arenal, Jérome Kalifa, Francisco Fernández- Avilés, and Omer Berenfeld, "Real-time dominant frequency mapping and ablation of dominant frequency sites in atrial fibrillation with left-to-right frequency gradients predicts long-term maintenance of sinus rhythm," Heart Rhythm , vol. 6, no. 1, pp. 33-40, Jan 2009.

[14] Raquel Cervigón, Javier Moreno, Jorge García-Quintanilla, Julián Pérez-Villacastín, and Francisco Castells, “Entropy at the right atrium as a predictor of atrial fibrillation recurrence outcome after pulmonary vein ablation," Biomed Tech (Berl) Jun 2015.

[15] Raquel Cervigón, Javier Moreno, Richard B. Reilly, José Millet, Julián Pérez-Villacastín, and FranciscoCastells. "Entropy measurements in paroxismal and persistent atrial fibrillation”. Physiol Meas, 31(7):1011-20, Jul 2010.

[16] J Jalife, "Spatial and temporal organization in ventricular fibrillation,” Trends Cardiovasc Med, vol. 9, no. 5, pp. 119-27, Jul 1999.

Address for correspondence:

Raquel Cervigón Abad

Escuela Politécnica. Camino del Pozuelo sn. Cuenca. Spain raquel.cervigon@uclm.es 